\title{
Droplet Microfluidic System with On-Demand Trapping and Releasing of Droplet for Drug Screening Applications
}

\author{
Matthew Courtney, ${ }^{\dagger}$ Xiaoming Chen, ${ }^{\ddagger}$ Sarah Chan, ${ }^{\dagger}$ Tarek Mohamed, ${ }^{\S}$ Praveen P. N. Rao, ${ }^{\S \odot}$ \\ and Carolyn L. Ren $*$ \\ 'Department of Nanotechnology Engineering, University of Waterloo, 200 University Avenue West, Waterloo, Canada \\ ${ }^{*}$ Department of Mechanical and Mechatronics Engineering, University of Waterloo, 200 University Avenue West, Waterloo, Canada \\ ${ }^{\S}$ School of Pharmacy, Health Sciences Campus, University of Waterloo, 200 University Avenue West, Waterloo, Canada
}

ABSTRACT: 96-Well plate has been the traditional method used for screening drug compounds libraries for potential bioactivity. Although this method has been proven successful in testing dose-response analysis, the microliter consumption of expensive reagents and hours of reaction and analysis time call for innovative methods for improvements. This work demonstrates a droplet microfluidic platform that has the potential to significantly reduce the reagent consumption and shorten the reaction and analysis time by utilizing nanolitersized droplets as a replacement of wells. This platform is evaluated by applying it to screen drug compounds that inhibit the tau-peptide aggregation, a phenomena related to Alzheimer's disease. In this platform, sample reagents are first dispersed into nanolitre-sized droplets by an immiscible carrier oil and then these droplets are trapped on demand in the downstream of the microfluidic device. The relative decrease in fluorescence through drug inhibition is characterized using an inverted epifluorescence. Finally, the trapped droplets are released on-demand after each test by manipulating the applied pressures to the channel network which allows continuous processing. The testing results agree well with that obtained from 96-well plates with mush lower sample consumption ( 200 times lower than 96-well plate) and reduced reaction time due to increased surface volume ratio ( $2.5 \mathrm{~min}$ vs $2 \mathrm{~h}$ ).

$\mathrm{T}$ raditional drug screening efforts rely on the use of 96- or 384-well plates to assess the efficacy of various drug candidates against key biological targets. ${ }^{1-6}$ For this process, setup typically consists of manual pipetting or robotic handlers to dispense a combination of reagents into each well. ${ }^{2,6-9}$ To add to the complications of handling this wide array of reagents, these platforms require large aliquots of expensive biological components and reagents, adding to the overall costs of the screening process. Furthermore, the screening process for each compound may take several hours to complete which can be time-consuming. Droplet microfluidics which utilizes monodispersed nanoliter-sized droplets that can be generated in microchannel networks at $\mathrm{kHz}$ rates to serve as reaction vesicles has the potential to address these problems. ${ }^{9,10}$ In general, droplets are produced by injecting one fluid into another immiscible fluid resulting water-in-oil or oil-in-water emulsions. ${ }^{11}$ For screening drug compounds, sample reagents can be dispersed into droplets by an immiscible oil and the drop volume can be varied from picoliter to nanoliter by tuning the operating and geometric conditions such as the applied pressures, flow rates, and channel dimensions Almost instantaneous mixing can be achieved due to the threedimensional flow nature within the droplets and large surface to volume ratio which eliminates the need for shaking the reagents to enhance mixing. ${ }^{12}$ These benefits are particularly useful for drug screening applications.

This study aims to explore the potential of employing droplet microfluidic platform as an alternative to screen drug compounds that inhibit the tau-peptide aggregation, a phenomenon related to Alzheimer's disease. Tau proteins stem from soluble monomeric peptides that aggregate to form large and insoluble cross- $\beta$-sheet structures. ${ }^{13-17}$ These aggregates lead to the neuronal cell death that is one of the hallmarks of Alzheimer's disease. ${ }^{13-17}$ To develop molecules which can prevent tau aggregation, the hexapeptide AcVQIVYK- $\mathrm{NH}_{2}$ (AcPHF6) which is derived from the full length of tau protein can be used to screen compound libraries for potential antitau aggregation properties ${ }^{17}$ and a fluorescent indicator known as thioflavin-T (ThT) is often used to quantify the aggregation. ${ }^{18}$ This is made possible by the fact that the fluorescence excitation and emission peaks of the ThT molecules shift to higher wavelengths when they bind to the cross- $\beta$-sheet structures. ${ }^{18}$ Therefore, as tau-peptide aggrega- 


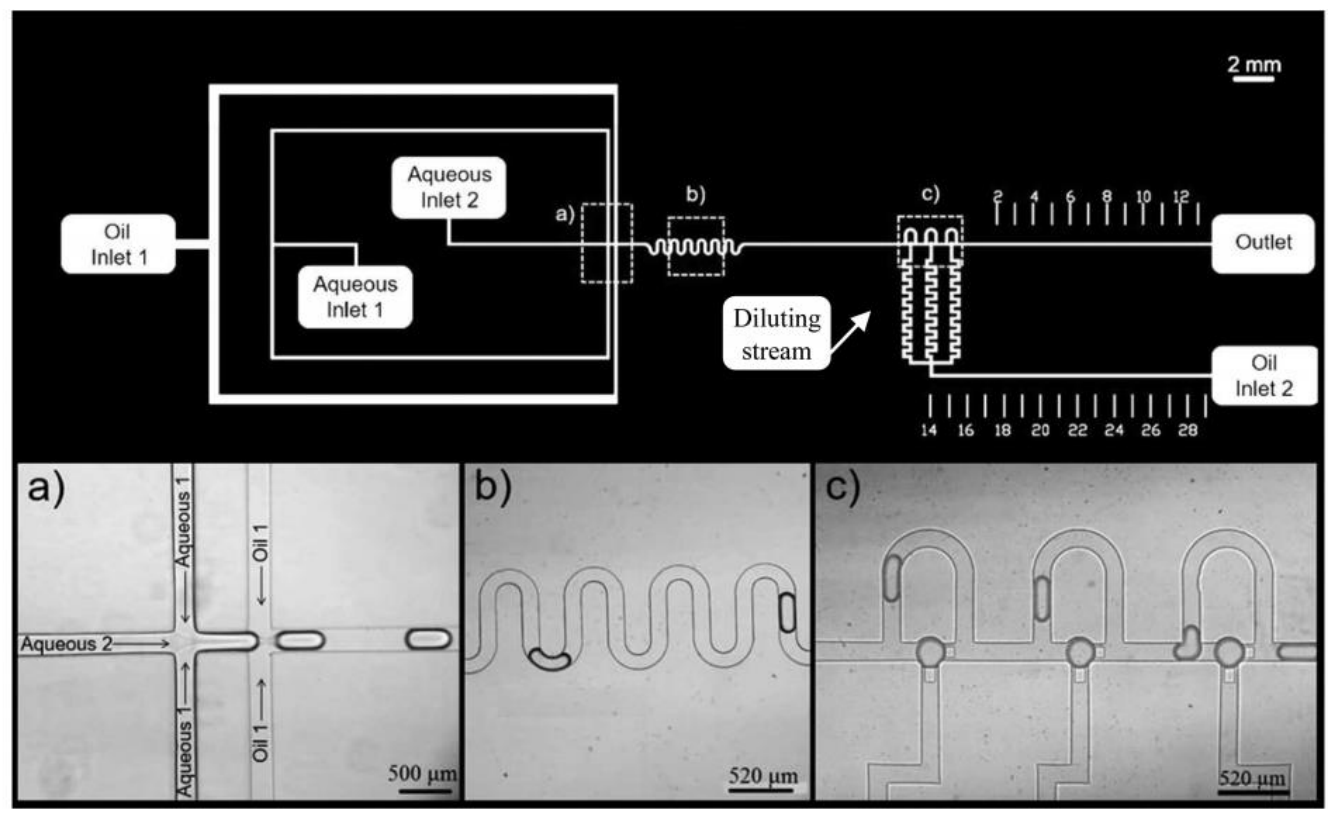

Figure 1. Sketch of the microfluidic design. Oil inlet 1 is for the continuous phase. Aqueous inlets 1 and 2 are where two separate dispersed phases can be injected. Oil inlet 2 is the diluting stream that controls the on-demand droplet trapping and releasing function. Region a shows droplets being generated from the two aqueous streams being mixed. Region b shows a series of serpentine channels that help mix contents within the droplets. Region $\mathrm{c}$ shows the droplet traps which are connected to the diluting stream.

tion increases, so does the fluorescence intensity. The presence of inhibitors helps to reduce tau-aggregation which should be quantified as an overall decrease in fluorescence intensity.

In our previous work, the 96-well plate format has been used for an AcPHF6 aggregation kinetics assay. ${ }^{17}$ The volume of each well was $200 \mu \mathrm{L}$ and the screening process usually took 2 $\mathrm{h}$ to complete. ${ }^{17} \mathrm{By}$ switching to a droplet-based platform in the nanoliter range, the sample volume is magnitudes lower than well plate systems, and reaction times can be reduced significantly because of the enhanced mixing resulted from the three-dimensional flow within the droplet. Premixing multiple reagents and then immediately encapsulating the mixture in droplets for reaction is possible in principle, however, not practical because reaction occurs within $30 \mathrm{~s}$ and completes around 2-3 $\mathrm{min}$ in droplets which requires precise control of the reaction start time. In addition, repeating experiments would require repeating the entire processing including mixing the reagents freshly. Therefore, designing a microchannel network with on-demand trapping and releasing functions is necessary because the reaction start time can be well controlled and repeating experiments is enabled by simply releasing the previously trapped droplets and starting with the newly trapped droplets. There are several existing methods capable of trapping and releasing on-demand, however, most involve complex active techniques such as electrodes ${ }^{19}$ and laser-induced heating. ${ }^{20}$

The study instead relies on passive methods to achieve rapid mixing within droplets, on-demand trapping and releasing of droplets. It is similar to a passive droplet trapping technique that breaks off parts of a long aqueous plug and leaves them trapped in individual wells. ${ }^{21,22}$ The major differences include the design of diluting streams (see Figure 1) that are connected to the series of the trapping wells to enable on-demand trapping and releasing and individual droplets can be trapped rather than pieces of a long plug. Both of these added features make on-chip mixing possible while facilitating rapid AcPHF6 aggregation feedback. The on-chip mixing was achieved using a stratified flow technique that combined an AcPHF6 solution with a solution of sodium phosphate dibasic heptahydrate (PBS buffer), ThT, and a proven tau-aggregation inhibitor known as orange $\mathrm{G}$ as the reference compound. ${ }^{17}$ Droplets were generated and mixed from these combined aqueous streams before being trapped seconds later. The pressure applied at the diluting stream controlled the trapping and releasing of droplets on-demand. While the droplets were being stored, ThT was used to monitor the tau-aggregation in the presence of orange $\mathrm{G}$ at various concentrations. The inhibitor results were then compared to positive and negative controls. Overall, the fluorescence intensity data obtained showed similar trends to that of a 96-well plate study. The volume of each trapped droplet was approximately $1 \mathrm{~nL}$, which is a significant volume reduction on the order of $10^{5}$ compared to one well volume of $200 \mu \mathrm{L}$. However, the total volume consumption including the sample wasted in the reservoir and tubing and the sample used for experiments $(\sim 1000$ droplets for one reaction repeating five times) is around $5 \mu \mathrm{L}$ for the droplet platform which is around 200 times lower than that of the well plate system which is around $1 \mathrm{~mL}$ for a reaction repeating five times. The screening process was completed in only $2.5 \mathrm{~min}$ because of the fast mixing time within the droplets. Finally, the droplets are trapped approximately $3 \mathrm{~s}$ after the AcPHF6 first comes into contact with the PBS buffer and other reagents. This is a major improvement from the 96-well plate setup which is timeconsuming, expensive and does not offer immediate fluorescence measurements after the aggregation process begins.

\section{EXPERIMENTAL SECTION}

Device Fabrication. The polydimethylsiloxane (PDMS) microfluidic devices were prepared using standard softlithography techniques. Briefly, the PDMS (Skylard 184, Dow 
Corning) is mixed in a 10:1 ratio of base to curing agent. The PDMS is poured onto a silicon master that is coated with SU-8 (2025, Microchem) microchannel patterns and cured for $3 \mathrm{~h}$ at $95^{\circ} \mathrm{C}$. The PDMS mold is then peeled off from the master and fluidic inlets and outlets are made using a $1.5 \mathrm{~mm}$ biopsy punch. This mold is bonded to a PDMS coated glass slide by exposing the two substrates to oxygen plasma (PDC-001, Harrick Plasma) with a power of $29.6 \mathrm{~W}$ at $500 \mathrm{mT}$ Torr for $10 \mathrm{~s}$. Finally, the PDMS devices are heated to $190^{\circ} \mathrm{C}$ for at least 2 days to ensure that the microchannels are hydrophobic.

Materials. Five cSt silicone oil (Sigma-Aldrich) was used as the carrier oil. An AcPHF6 (Celtek Peptides) stock solution was freshly prepared using ultrapure water (Cayman Chemical Company) at a concentration of $0.12 \mathrm{mg} / \mathrm{mL}$. The AcPHF6 had a minimum purity of $98 \%$. A $50 \mathrm{mM}$ PBS buffer (SigmaAldrich) stock solution was prepared using ultrapure water and the $\mathrm{pH}$ was adjusted to 7.3 using $\mathrm{HCl}$. A $1.57 \mathrm{mM}$ ThT (Sigma-Aldrich) stock solution was freshly prepared with PBS buffer and its glassware was wrapped in foil to protect the light sensitive dye. A $500 \mu \mathrm{M}$ orange G (Sigma-Aldrich) solution was prepared with PBS buffer and $5 \%(\mathrm{v} / \mathrm{v})$ dimethyl sulfoxide(DMSO). The DMSO (Sigma-Aldrich) was used to help dissolve the orange $\mathrm{G}$ in solution. No surfactants were added for any reaction because they may influence the assay and reduce the trapping performance because of the reduced interfacial tension.

Experimental Setup. To test the on-demand droplet trapping and releasing capabilities of the microfluidic device, fluids were pumped into the inlets using a high precision microfluidic pressure control system (MSFC 8C, Fluigent). The flow rate of the dispersed phase was measured using a flow sensor (SLG 1430, Sensirion). The sample reagents were injected into the microfluidic device using fluidic tubing with inner diameter of $100 \mu \mathrm{m}$ (IDEX Health \& Science). The droplet behavior was imaged using an inverted epifluorescence microscope (ECLIPSE Ti, Nikon) with a $4 \times$ objective and a high speed camera (Phantom v210, Vision Research). Each video was captured at 75 frames per second.

Droplet Trapping and Releasing On-demand. A microfluidic device capable of mixing, trapping, and releasing droplets on-demand was designed. An overall layout of the microchannel network is shown in Figure 1. Controlling the concentration of a particular reagent such as the inhibitor in individual droplets is important for drug screening analysis which is designed to achieve by varying the reagent concentrations in the carrier aqueous streams such as Aqueous stream 1 and 2 and the flow rate ratio of these two streams. For added versatility, aqueous inlet 1 can be divided into two inlets by punching two reservoirs along the two stream paths allowing for a third aqueous stream from inlet 2 . By pumping only water into aqueous inlet 2 , the middle stream can then act as a diluter, or a barrier that keeps contents from the other two streams separated prior to droplet generation. In either case, the contents from each aqueous stream can be mixed through a series of serpentine channels as seen in Figure $1 \mathrm{~b}$ and then trapped shown in Figure 1c. The trapped droplets remain stationary in the circular traps which increase the hydrodynamic resistance so that other droplets continue flowing through the main channel. The bottom of each trap is connected to the diluting stream, and the oil flow through this stream comes from oil inlet 2 as shown in Figure 1. The pressure system can be used to precisely control the oil flow through the diluting stream. There are two pillars in each trap, and these are what allows droplets to remain stationary in the traps, as it prevents the droplet from moving forward.

To ensure droplets are trapped on-demand, the following criteria must be met: (1) when a trap is empty, the approaching droplet can be trapped or forced to go through the bypass channel for on-demand trapping; (2) when a trap is filled with one droplet, no more droplets should be trapped; and (3) when a droplet is trapped, it should not be pushed out the trap unless on-demand. To meet these criteria, the flow rate ratio between the bypass channel and the trap path $\left(Q_{B} / Q_{T}\right)$ must be well controlled because a droplet at the entrance of the trap always follows the stream with a higher flow rate. For example, if $Q_{B}>$ $Q_{T}$, the droplet will go through the bypass channel as demonstrated in Figure $2 \mathrm{~b}$, vise versa as shown in Figure $2 \mathrm{c}$.

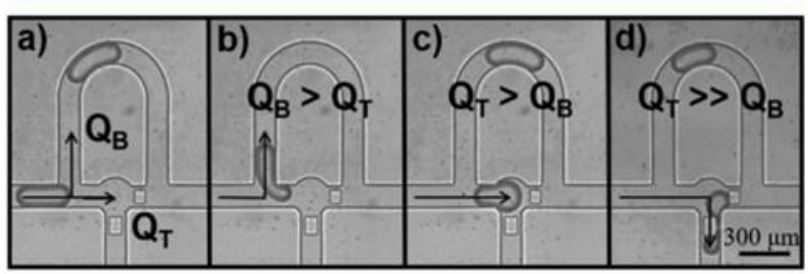

Figure 2. Droplet trapping scenarios. Image a shows flow rates $Q_{B}$ and $Q_{T}$. Image b shows the scenario where droplets enter the bypass channel rather than the trap. Image $c$ shows the scenario, where a droplet enters the trap. Image $d$ shows the scenario where enters the trap and pushes through to the diluting stream.

There is a chance that $Q_{T} \gg Q_{B}$ which causes the trapped droplet to be pushed through the pillar unwantedly as seen in Figure $2 \mathrm{~d}$. Therefore, by fine-tuning $Q_{T}$ in relevance to $Q_{B}$, a droplet can be trapped on-demand as shown in Figure $2 \mathrm{c}$ and also released on-demand as shown in Figure $2 \mathrm{~b}$.

The ratio of $Q_{T}$ and $Q_{B}$ is influenced by several parameters such as channel dimensions, the applied pressures in reservoirs and the trap status (i.e., with/out droplets). If the pressure at the Oil Inlet 2 is set to be 0 , the trapping is mainly influenced by channel dimensions and the applied pressures which are coupled together through the hydraulic resistance $\left(R_{\mathrm{H}}\right)$ of the microchannel ${ }^{23}$ as seen in eq $1^{23}$ where $\Delta P$ is the pressure drop over the channel length:

$$
\Delta P=Q R_{\mathrm{H}}
$$

To trap a droplet as shown in Figure 2c, the lengths of the bypass channel $\left(L_{\mathrm{B}}\right)$ and the outlet channel $\left(L_{\mathrm{O}}\right)$ (the distance between the last trap and the outlet), which are proportional to their RHs, respectively, were designed, such that the $Q_{T} / Q_{B}$ ratio was slightly greater than 1 for all three traps as shown in Figure $3 . L_{\mathrm{O}}$ can be varied by simply changing the location of where the outlet is punched. For this design, the outlet channel was $6 \mathrm{~mm}$ and the channel height $(h) 25 \mu \mathrm{m}$. The diameter of the trap $\left(d_{\mathrm{T}}\right)$ was chosen to be $240 \mu \mathrm{m}$ to allow for droplets to fill the total volume while having a sufficient hydraulic resistance for trapping and releasing. The length of each diluting stream $\left(L_{\mathrm{D}}\right)$ was chosen to be $30 \mathrm{~mm}$. This large dimension was chosen to increase hydraulic resistance and limit the flow into the diluting stream during trapping. In doing so, trapping efficiency is influenced primarily by the $Q_{T} / Q_{B}$ ratio.

To avoid the trapped droplet to be pushed through the pillars as seen in Figure $2 \mathrm{~d}$, the pressure drop across the trapped droplet should be below a critical pressure drop $\left(\Delta P_{c}\right)$, 


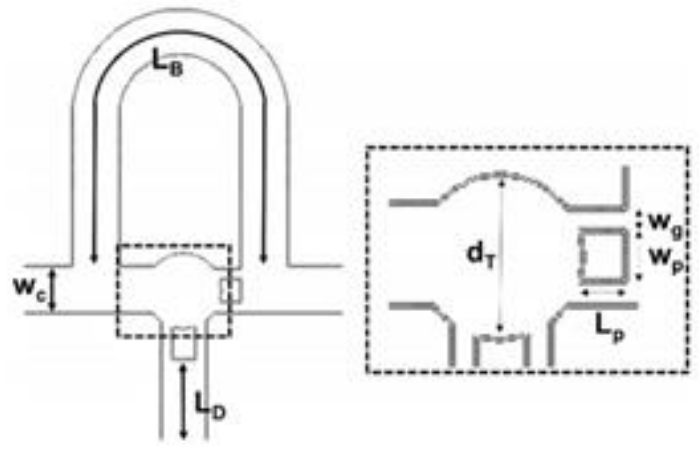

Figure 3. Microchannel dimensions for the trapping design. The box with dashed outlines shows a close-up view of the trap dimensions. $L_{\mathrm{B}}$ $=1.8 \mathrm{~mm}, L_{\mathrm{D}}=30 \mathrm{~mm}, w_{\mathrm{c}}=150 \mu \mathrm{m}, d_{\mathrm{r}}=240 \mu \mathrm{m}, w_{\mathrm{g}}=25 \mu \mathrm{m}, w_{\mathrm{p}}=$ $73 \mu \mathrm{m}, L_{\mathrm{p}}=70 \mu \mathrm{m}$.

which can be estimated with the Young-Laplace relation seen in eq 2 , where $\gamma$ is the interfacial tension. ${ }^{24}$

$$
\Delta P_{c}=\gamma\left(\frac{2}{h}+\frac{2}{w_{g}}\right)
$$

As the flow rate of $Q_{T}$ increases, the pressure drop across the droplet tends to be greater than $\Delta P_{\sigma}$ and thus push the droplet through the pillars. Furthermore, due to this $\Delta P_{c}$ criteria, having a higher hydraulic resistance in the microchannel means that droplet trapping will fail at lower flow rates. Therefore, low viscosity silicon oil (5cSt) was used as the carrier oil which results in low $\mathrm{RH}$, and thus allows for a higher speed droplet to be trapped. As seen in eq 2 , reducing values of $h$ and $w_{g}$ can also add tolerance for higher pressure drop across the trapped droplet, however, they are limited by fabrication techniques and tend to be closed due to PDMS swelling. For this design, $w_{8}$ was optimized to be $25 \mu \mathrm{m}$ to allow droplets to be trapped efficiently. For larger $w_{g}$ values, droplets had difficulty remaining in the trap because of the large gaps and a small $\Delta P_{c}$ value. Additionally, with this $w_{g}$ value in mind, $h$ was limited to $25 \mu \mathrm{m}$ because of the fabrication limit as well.

For a given set of channel dimensions, varying the applied pressure at the oil inlet 2 is effective in tuning $Q_{T}$ especially when releasing on-demand is desired because increasing this pressure increases the flow rate of the diluting stream (equivalent to decreasing $Q_{\mathrm{r}}$ ) allowing a trapped droplet to be pushed out the trap and through the bypass channel. The diluting stream pressure $\left(P_{\mathrm{DS}}\right)$ required to release the droplets was 900 mbar. Figure 4 illustrates the trapping and releasing on-demand process when the droplet speed was $1.9 \mathrm{~mm} / \mathrm{s}$. The dynamic trapping and releasing processes are shown in Supporting Information Video 1 and 2, respectively. It is not a requirement for $P_{\mathrm{DS}}$ to be set to 0 mbar to trap droplets as it can be increased slightly to improve trapping at higher flow rates. Overall, the on-demand trapping function works for a wide range of flow rates and the droplets can then be released by simply increasing the diluting stream pressure.

AcPHF6 Aggregation Kinetics Assay. The on-demand trapping and releasing function was used to monitor tau aggregation in the presence of an inhibitor known as orange G. For this assay, the aqueous phases were pumped into the microfluidic device using a dual syringe pump (Pump33, Harvard Apparatus). 5eSt silicone oil was injected into the chip using the pressure control system. After one droplet was

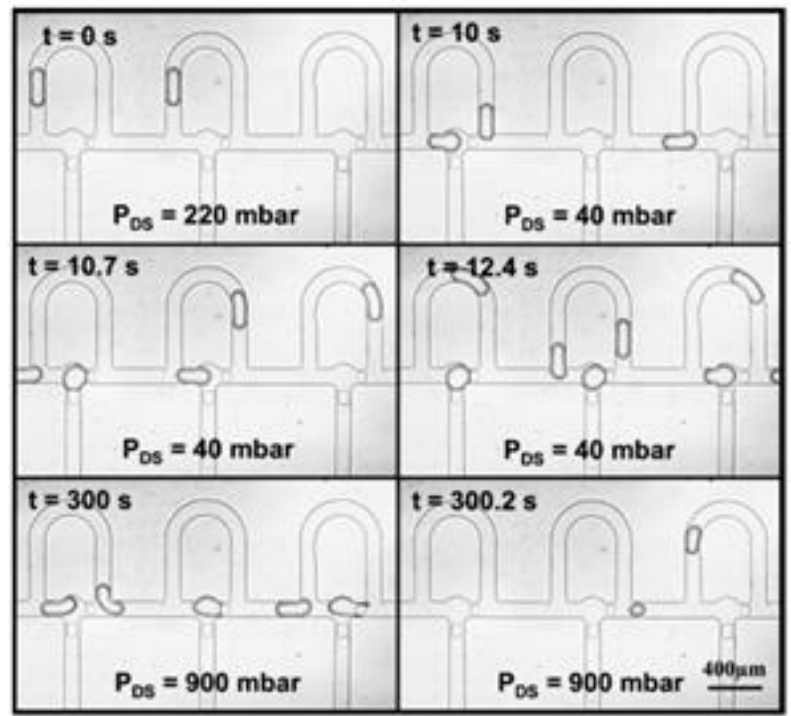

Figure 4. On-demand trapping and releasing process. Here, $P_{\mathrm{DS}}$ is initially at $220 \mathrm{mbar}$ to ensure no droplets enter the traps. Then, $P_{\mathrm{DS}}$ is reduced to 40 mbar to allow droplets enter all three traps in approximately $2.4 \mathrm{~s}$. The droplets are stored in the traps for $5 \mathrm{~min}$ before changing $P_{\mathrm{DS}}$ to 900 mbar to release the droplets.

trapped at the last well (the third from left in Figure 1), the aggregation process was imaged using the inverted epifluorescence microscope and a high sensitivity CCD Camera (Retiga $2000 \mathrm{R}$, QImaging). A 40x objective was used to image each trapped droplet in order to eliminate any background noise from the PDMS microchannels. Illumination was supplied by a $100 \mathrm{~W}$ mercury halide lamp (Intensilight C-HGFIE, Nikon). A filter cube (CFP-HQ Nikon) was used to image the ThT fluorescence (440 nm excitation and $490 \mathrm{~nm}$ emission). The hardware gain was 21.5 and the exposure time was set to 100 ms. After about $5 \mathrm{~min}$ of imaging the droplets, they were released and the traps were reused for more tests. The fluorescence intensity for each droplet was measured using an imaging software (NIS-Elements AR, Nikon). The percent change in fluorescence intensity was calculated for each droplet to compare them to one another.

There were two concentrations of orange G used for this study, $6 \mu \mathrm{M}$ and $16.5 \mu \mathrm{M}$. They were both compared to positive and negative controls to help determine the effectiveness of orange $\mathrm{G}$ as a tau-peptide aggregation inhibitor. For each test, the approximate final droplet concentrations are summarized in Table 1 . Originally, the droplet concentrations were such that the final ThT concentration was $8.25 \mu \mathrm{M}$, to replicate the 96-well plate study. This concentration proved to

Table 1. Final Concentrations of Each Compound after a Droplet Gets Trapped

$\begin{array}{cccccc}\text { test } & \begin{array}{c}\text { AcPHF6 } \\ (\mathrm{mg} / \mathrm{mL})\end{array} & \begin{array}{c}\text { ThT } \\ (\mu \mathrm{M})\end{array} & \begin{array}{c}\text { orange G } \\ (\mu \mathrm{M})\end{array} & \begin{array}{c}\text { PBS buffer } \\ (\mathrm{mM})\end{array} & \begin{array}{c}\text { DMSO }(\% \\ (\mathrm{v} / \mathrm{v}))\end{array} \\ \begin{array}{c}\text { positive } \\ \text { control }\end{array} & 0.03 & 3.1 & & 16.3 & 0.33 \\ \begin{array}{c}\text { orange G } \\ (6 \mu \mathrm{M})\end{array} & 0.03 & 3.1 & 6 & 16.4 & 0.12 \\ \begin{array}{c}\text { orange } \mathrm{G} \\ (165 \mu \mathrm{M})\end{array} & 0.03 & 3.1 & 16.5 & 16.3 & 0.33 \\ \begin{array}{c}\text { negative } \\ \text { control }\end{array} & & 3.1 & & 16.3 & 0.33 \\ & & & & & \end{array}$


be too high for droplets as the ThT molecules were suspected of self-aggregating and caused an unwanted increase in fluorescence intensity for the negative control droplets. Therefore, the ThT concentration was reduced until there was no significant increase in the negative control fluorescence intensity. The concentrations of all other reagents were decreased proportionally to the ThT concentration. An added benefit of this protocol was the reduction in the AcPHF6 concentration that significantly reduced its aggregation at the droplet generator, where the peptide solution first comes into contact with the PBS buffer.

For the negative control, a single syringe was prepared with the same reagent concentrations shown in Table 1 . It was connected to aqueous inlet 1 , and aqueous inlet 2 was blocked. For the positive control and inhibitor tests, two syringes were used for the aqueous phases. The AcPHF6 stock solution was kept in its own syringe to prevent its aggregation prior to droplet generation. This syringe was connected to aqueous inlet 2 , and the flow rate was set to $0.5 \mu \mathrm{L} / \mathrm{min}$. The second syringe was connected to aqueous inlet 1 , and the flow rate was set to $1.5 \mu \mathrm{L} / \mathrm{min}$. For the positive control, it contained $4.13 \mu \mathrm{M}$ of ThT, $21.7 \mathrm{mM}$ of PBS buffer, and $0.44 \%$ (v/v) DMSO. For the $6 \mu \mathrm{M}$ and $16.5 \mu \mathrm{M}$ orange $\mathrm{G}$ tests, it contained similar concentrations as the positive control with the addition of 8 $\mu \mathrm{M}$ and $22 \mu \mathrm{M}$ orange $\mathrm{G}$, respectively. With the flow rates of the first and second syringe at a 1:3 ratio, the final droplet concentrations in Table 1 can be achieved. Additionally, the syringes were kept on ice for the length of the tests.

\section{RESULTS AND DISCUSSION}

The effectiveness of orange $\mathrm{G}$ as an AcPHF6 aggregation inhibitor was determined by comparing the fluorescence intensity change at two different concentrations with positive and negative controls. Figure 5 shows the results for this drug screening study. These intensity curves are all averaged from at least three different droplets that were measured over a $5 \mathrm{~min}$ period. As expected, the positive control curve had the greatest change in intensity and the negative control curve did not change in intensity. This trend is consistent with the result from

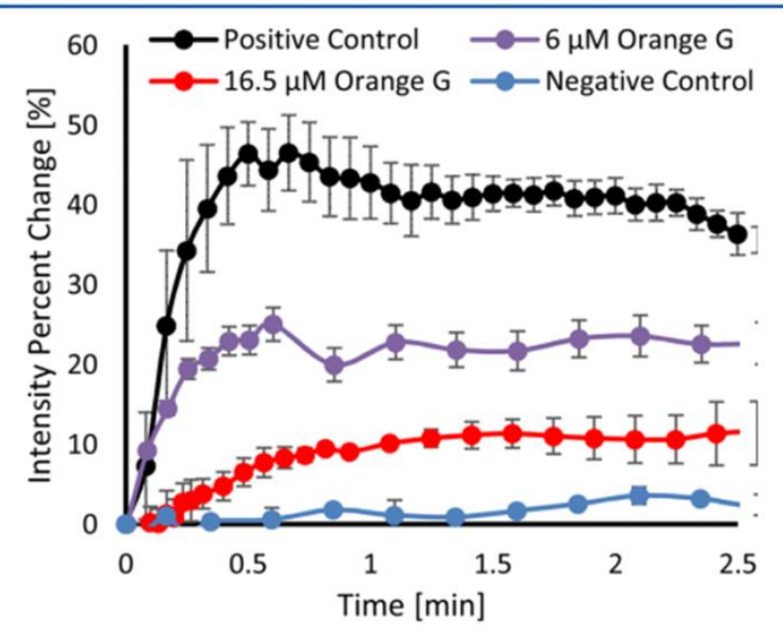

Figure 5. Plot representing the AcPHF6 aggregation over time in the presence of the orange $\mathrm{G}$ inhibitor. The positive control curve has the highest percent change in intensity, followed by $6 \mu \mathrm{M}$ orange $\mathrm{G}$ and then $16.5 \mu \mathrm{M}$ orange $\mathrm{G}$. There is no significant change in intensity for the negative control curve. the 96-well plate format indicating the rapid aggregation of AcPHF6 in the absence of an inhibitor. ${ }^{17}$ Additionally, both orange $\mathrm{G}$ curves at 6 and $16.5 \mu \mathrm{M}$ experienced a change in intensity which was between the positive and negative control curves. This indicates that AcPHF6 aggregation was reduced in the presence of orange G. Furthermore, at $6 \mu \mathrm{M}$, orange $\mathrm{G}$ concentration has a greater change in fluorescence intensity than that of the 16.5 orange $G$ concentration, which makes sense given that there is less inhibitor to reduce aggregation. Both of these outcomes demonstrate that this droplet microfluidic platform has the potential to be used as an alternative drug screening tool for rapid analysis of large compound libraries.

The shape of the curves in Figure 5 is comparable to the trends seen in the 96-well plate study. ${ }^{17}$ The most notable similarity is that there is a rapid increase in fluorescence before reaching a plateau phase. This rapid increase represents the AcPHF6 aggregation in the reaction vessel. In the 96-well plate, it takes around $30 \mathrm{~min}$ for the AcPHF6 aggregation to plateau. This is greater than what is seen in Figure 5, as the AcPHF6 aggregation takes roughly $30 \mathrm{~s}$ to plateau in the trapped droplets. The significant decrease in reaction time can be attributed to the rapid mixing of reagents inside the droplets. The volume of the droplets can be approximated by treating the trap as a cylinder. With a channel height of $25 \mu \mathrm{m}$ and a trap diameter of $240 \mu \mathrm{m}$, the droplet volume works out to be about $1.13 \mathrm{~nL}$. Given that the volume for each well in a 96-well plate is $200 \mu \mathrm{L}$, switching to a droplet microfluidics platform demonstrates a significant volume reduction, in addition to significant reduction in the time required for screening.

The fluorescent images in Figure 6 show examples of droplets over time for each test. The positive control droplets

\begin{tabular}{|c|c|c|c|c|c|}
\hline & $0 \mathrm{~s}$ & $15 \mathrm{~s}$ & $30 \mathrm{~s}$ & $60 \mathrm{~s}$ & $120 \mathrm{~s}$ \\
\hline \multicolumn{6}{|l|}{ Positive } \\
\hline \multicolumn{6}{|l|}{$\begin{array}{c}6 \mu \mathrm{M} \\
\text { Orange G }\end{array}$} \\
\hline $\begin{array}{c}16.5 \mu \mathrm{M} \\
\text { Orange } \mathrm{G}\end{array}$ & , & z & t & & . \\
\hline Negative & & & & & \\
\hline Control & & & & & $100 \mu \mathrm{m}$ \\
\hline
\end{tabular}

Figure 6. Trapped droplets with fluorescence intensity changing over time. Fluorescent images for positive control, orange $\mathrm{G}$ at $6 \mu \mathrm{M}$ and $16.5 \mu \mathrm{M}$, and negative control droplets can be seen after $0,15,30$, and $120 \mathrm{~s}$. The positive control droplet has the most noticeable change in fluorescence. The fluorescence change for both orange $\mathrm{G}$ droplets is subtle. Finally, there is no noticeable change in fluorescence for negative control.

have a much stronger increase in fluorescence when compared to the droplets containing orange $\mathrm{G}$, and this is in compliance with the curves seen in Figure 5. The droplets containing AcPHF6 all exhibit some fluorescence at the initial measurement. This is because of the aggregation that takes place between the droplet generator and the final trapping well, which is around $30.26 \mathrm{~mm}$ in distance. With a velocity of approximately $8.89 \mathrm{~mm} / \mathrm{s}$, it takes about $3.4 \mathrm{~s}$ for the droplet to reach the trap after the AcPHF6 solution makes contact with 
the PBS buffer at the droplet generator. Considering the time scale in Figure 5, missing the first few seconds of aggregation will not have a significant impact on the fluorescence measurements.

The trapped droplets were generated using a stratified flow technique that mixed reagents seconds before measuring the fluorescence intensity. This is a cost-effective alternative to the robotic liquid handlers that inject reagents into each well of the 96-well plate format. There is still some error that exists in the stratified flow technique that may lead to some of the error bars seen in Figure 5. For one, there are some fluctuations in the flow rates from the syringe pumps which can lead to a nonuniform reagent concentration from one droplet to the next. Additionally, unlike the 96-well plate, the fluids within each syringe cannot be mixed during the tests and this may lead to uneven reagent distribution over time. Another source of error stems from the fact that this design does not allow droplets to be screened in parallel. Instead, only one type of droplet can be screened at a time. In the future, it is recommended that the design be modified to trap one test compound in parallel with both controls. In doing so, this will allow for more rigorous comparisons and lead to more accurate AcPHF6 aggregation data. Nonetheless, this on-demand trapping and releasing mechanism shows the potential for using droplet microfluidics as a cost-effective alternative to the 96-well plate format which can be used for rapid screening to test compound libraries.

\section{CONCLUSION}

A microfluidic device capable of mixing, trapping, and releasing droplets on-demand was designed and fabricated using standard soft-lithography techniques. It demonstrated the ability to trap droplets in a short period of time at high droplet speeds. The device was used in an AcPHF6 tau-hexapeptide aggregation kinetics assay to explore the potential of droplet microfluidics as an alternative to the 96-well plate drug screening platform. A noticeable decrease in AcPHF6 aggregation was detected when testing a proven inhibitor known as orange $\mathrm{G}$. When comparing it to the 96-well plate study, the reaction volume was reduced by a factor of $10^{2}$ and the reactions times were reduced from 2 $\mathrm{h}$ to $2.5 \mathrm{~min}$. Additionally, the on-chip mixing, rapid fluorescence detection, and on-demand droplet releasing helps facilitate an easier setup procedure for users. In essence, this study demonstrated that a microfluidic device capable of trapping and releasing droplets on-demand offers several advantages over the traditional 96-well plate platform for drug screening applications.

\section{Notes}

The authors declare no competing financial interest.

\section{ACKNOWLEDGMENTS}

The authors acknowledge the Natural Science and Engineering Research Council of Canada, Canada Research Chair program, Canada Foundation for Innovation, and University of Waterloo for research grants to C.L.R., the Ontario Mental Health Foundation for a $\mathrm{PhD}$ studentship to T.M., NSERC-Discovery (RGPIN 03830-2014) and the Ministry of Research and Innovation, Government of Ontario, Canada for an Early Research Award to P.P.R.

\section{REFERENCES}

(1) Pereira, D.; Williams, J. Br. J. Pharmacol. 2007, 152, 53-61.

(2) Reid, B.; Stratton, M.; Bowers, S.; Cavasin, M.; Demos-Davies,

K.; Susano, I.; McKinsey, T. J. Mol. Cell. Cardiol. 2016, 97, 106-113.

(3) Bader, C.; Jesudoss Chelladurai, J.; Thompson, K.; Hall, C.; Carlson, S.; Brewer, M. Vet. Parasitol. 2016, 223, 34-37.

(4) Lariosa-Willingham, K.; Rosler, E.; Tung, J.; Dugas, J.; Collins, T.; Leonoudakis, D. BMC Neurosci. 2016, 17, 1-13.

(5) Shi, X.; Sha, S.; Liu, L.; Li, X.; Ma, Y. Anal. Biochem. 2016, 498, 53-58.

(6) Lim, K.; Zahari, Z.; Amanah, A.; Zainuddin, Z.; Adenan, M. Exp. Parasitol. 2016, 162, 49-56.

(7) Mallet, C.; Lu, Z.; Fisk, R.; Mazzeo, J.; Neue, U. Rapid Commun. Mass Spectrom. 2003, 17, 163-170.

(8) Chen, D.; Ismagilov, R. Curr. Opin. Chem. Biol. 2006, 10, 226231.

(9) Dittrich, P.; Manz, A. Nat. Rev. Drug Discovery 2006, 5, 210-218.

(10) Neuži, P.; Giselbrecht, S.; Länge, K.; Huang, T.; Manz, A. Nat. Rev. Drug Discovery 2012, 11, 620-632.

(11) Holtze, C.; Rowat, A. C.; Agresti, J. J.; Hutchison, J. B.; Angilè, F. E.; Schmitz, C. H. J.; Köster, S.; Duan, H.; Humphry, K. J.; Scanga, R. A.; Johnson, J. S.; Pisignano, D.; Weitz, D. A. Lab Chip 2008, 8, 1632-1639.

(12) Teh, S. Y.; Lin, R.; Hung, L. H.; Lee, A. Lab Chip 2008, 8, 198220.

(13) Ross, C.; Poirier, M. Nat. Med. 2004, 10 (Suppl), S10-7.

(14) Ballatore, C.; Lee, V.; Trojanowski, J. Nat. Rev. Neurosci. 2007, $8,663-72$.

(15) Medeiros, R.; Baglietto-Vargas, D.; LaFerla, F. CNS Neurosci. Ther. 2011, 17, 514-524.

(16) von Bergen, M.; Barghorn, S.; Biernat, J.; Mandelkow, E.-M.; Mandelkow, E. Biochim. Biophys. Acta, Mol. Basis Dis. 2005, 1739, $158-166$.

(17) Mohamed, T.; Hoang, T.; Jelokhani-Niaraki, M.; Rao, P. ACS Chem. Neurosci. 2013, 4, 1559-1570.

(18) Hudson, S.; Ecroyd, H.; Kee, T.; Carver, J. FEBS J. 2009, 276, 5960-5972.

(19) Wang, W.; Yang, C.; Li, C. Lab Chip 2009, 9, 1504-6.

(20) Tan, W. H.; Takeuchi, S. Proc. Natl. Acad. Sci. U. S. A. 2007, 104, $1146-1151$.

(21) Dewan, A.; Kim, J.; McLean, R.; Vanapalli, S.; Karim, M. Biotechnol. Bioeng. 2012, 109, 2987-2996.

(22) Bithi, S.; Vanapalli, S. Biomicrofluidics 2010, 4, 044110.

(23) Oh, K.; Lee, K.; Ahn, B.; Furlani, E. Lab Chip 2012, 12, 515545 .

(24) Boukellal, H.; Selimović, S.; Jia, Y.; Cristobal, G.; Fraden, S. Lab Chip 2009, 9, 331-338. 